\title{
Quantum Entanglement: Spooky Action at a Distance
}

\author{
Stan Gudder \\ Department of Mathematics, University of Denver, Denver, Colorado, USA. E-mail: sgudder@du.edu \\ Editors: James F. Glazebrook \& Danko D. Georgiev \\ Article history: Submitted on June 2, 2020; Accepted on June 14, 2020; Published on June 17, 2020.
}

\section{Q}

uantum entanglement is an important resource in quantum information technologies. Here, we study and characterize in a precise mathematical language some of the weird and nonintuitive features of quantum entanglement. We begin by illustrating why entanglement implies action at a distance. We then introduce a simple criterion for determining when a pure quantum state is entangled. Finally, we present a measure for the amount of entanglement for a pure state.

Quanta 2020; 9: 1-6.

\section{Quantum Mechanics in a Nutshell}

Entanglement is an important concept in quantum theory and many scientists believe it is responsible for much of the weirdness and nonintuitive nature of this theory [1--3]. Albert Einstein called entanglement "spooky action at a distance" and there are many people who agree. As strange as it may be, entanglement is a useful resource and it is the underlying basis for the speed and power of quantum computation [3-6].

We begin with a nutshell summary of quantum mechanics. The basic framework consists of a complex Hilbert space $H$ with inner product $\langle\phi, \psi\rangle$ and the set of (bounded) linear operators $\mathcal{L}(H)$ on $H$. For simplicity, we shall as-

(c) This is an open access article distributed under the terms of the Creative Commons Attribution License CC-BY-3.0, which permits unrestricted use, distribution, and reproduction in any medium, provided the original author and source are credited. sume that $H$ is finite dimensional in which case $\mathcal{L}(H)$ is represented by a set of complex matrices. This is general enough to include the theory of quantum computation and information [3-7]. It also has the advantage of making this article accessible to anyone who has had a first course in linear algebra. In the sequel, when we discuss a quantum state, we shall mean a pure state. There is a more general concept of mixed states, but we only mention these briefly.

A simplified version of the main axioms of quantum mechanics are the following [3, 4, 7].

Axiom 1. The states of a quantum system are represented by unit vectors in $H$.

Axiom 2. The quantum events are represented by projections on $H$.

Axiom 3. If $\psi \in H$ is a state and $P \in \mathcal{L}(H)$ is a projection, then

$$
\mathcal{P}_{\psi}(P)=\langle\psi, P \psi\rangle
$$

is the probability that $P$ occurs in the state $\psi$ and if $P$ does occur, then $\psi$ is updated to the state

$$
\psi^{\prime}=\frac{P \psi}{\|P \psi\|}
$$

Axiom 4. If $H_{1}, H_{2}$ represent two interacting quantum systems, then the combined system is represented by the tensor product $\mathrm{H}_{1} \otimes \mathrm{H}_{2}$.

Axiom 5. If $P_{1}, P_{2}$ are events in system 1 and 2 , respectively, then $P_{1} \otimes I_{2}, I_{1} \otimes P_{2}$ are the corresponding events in the combined system, where $I_{1} \in \mathcal{L}\left(H_{1}\right), I_{2} \in \mathcal{L}\left(H_{2}\right)$ are the identity operators. 
We now briefly elaborate on these axioms. Quantum mechanics can be thought of as a generalized probability theory [2, 4, 7]. A unit vector $\psi \in H$ satisfies

$$
\|\psi\|^{2}=\langle\psi, \psi\rangle=1
$$

and $\psi$ gives a quantum probability measure in accordance with Axiom 3 Recall that $P \in \mathcal{L}(H)$ is a projection if $P^{2}=P=P^{*}$. Axiom 3 connects the abstract concepts of states and events to the outcomes of experiments in the laboratory. Quantum mechanics cannot make precise predictions, it can only produce probabilities for the occurrence of events. The zero operator 0 represents the event that never occurs and $I$ represents the event that always occurs. An event $P$ occurs if and only if its complement $P^{\prime}=I-P$ does not occur and

$$
\mathcal{P}_{\psi}\left(P^{\prime}\right)=\langle\psi,(I-P) \psi\rangle=\langle\psi, \psi\rangle-\langle\psi, P \psi\rangle=1-\mathcal{P}_{\psi}(P)
$$

The updating $\psi \mapsto \psi^{\prime}$ in Axiom 3 is sometimes called the "collapse" of the state upon performing a measurement. This corresponds to the "ontic" viewpoint in which $\psi$ is considered to be a real, physical object [2]. Another way of viewing this is that once the occurrence of $P$ is confirmed, this information gives a "more precise" state $\psi^{\prime}$. We then call $\psi^{\prime}$ the state $\psi$ conditioned on the occurrence of the event $P$, which is similar to a conditional probability of ordinary statistics. This corresponds to the "epistemic" viewpoint in which $\psi$ is not considered to be a physical object but is only a carrier of our knowledge of the system [2]. One can employ either of these philosophies and still get the same quantum predictions. Once we are in the state $\psi^{\prime}$, then $P$ must occur because Axiom 3 gives

$$
\begin{aligned}
\mathcal{P}_{\psi^{\prime}}(P) & =\left\langle\psi^{\prime}, P \psi^{\prime}\right\rangle=\frac{1}{\|P \psi\|^{2}}\left\langle P \psi, P^{2} \psi\right\rangle \\
& =\frac{1}{\|P \psi\|^{2}}\langle P \psi, P \psi\rangle=1
\end{aligned}
$$

Notice that there is no problem with dividing by $\|P \psi\|$ because if $P$ has occurred, then

$$
\|P \psi\|^{2}=\langle P \psi, P \psi\rangle=\langle\psi, P \psi\rangle=\mathcal{P}_{\psi}(P) \neq 0
$$

Also notice that since $P$ or $P^{\prime}$ must occur, the state $\psi$ is updated to $P \psi /\|P \psi\|$ or $P^{\prime} \psi /\left\|P^{\prime} \psi\right\|$ when $P$ is tested.

The tensor product $H_{1} \otimes H_{2}$ is an important way to combine two Hilbert spaces. If $\left\{\phi_{i}\right\},\left\{\psi_{j}\right\}$ are orthonormal bases for $H_{1}, H_{2}$, respectively, then by definition, any vector in $H_{1} \otimes H_{2}$ has the form

$$
\gamma=\sum_{i, j} c_{i j} \phi_{i} \otimes \psi_{j}, \quad c_{i j} \in \mathbb{C}
$$

The main properties of $H_{1} \otimes H_{2}$ are that $\phi \otimes \psi$ is linear in both arguments and that

$$
\left\langle\alpha_{1} \otimes \beta_{1}, \alpha_{2} \otimes \beta_{2}\right\rangle=\left\langle\alpha_{1}, \alpha_{2}\right\rangle\left\langle\beta_{1}, \beta_{2}\right\rangle
$$

It follows that $\gamma$ in (1) is a state if and only if $\sum\left|c_{i j}\right|^{2}=1$. If $A \in \mathcal{L}\left(H_{1}\right), B \in \mathcal{L}\left(H_{2}\right)$ then $A \otimes B \in \mathcal{L}\left(H_{1} \otimes H_{2}\right)$ and

$$
A \otimes B(\alpha \otimes \beta)=A \alpha \otimes B \beta
$$

and any operator on $H_{1} \otimes H_{2}$ has the form $\sum c_{i j} A_{i} \otimes B_{j}$, $c_{i j} \in \mathbb{C}$. If $P_{1}$ is an event in system 1 , then $P_{1} \otimes I_{2}$ is the corresponding event in the combined system because $P_{1} \otimes I_{2}$ occurs in $H_{1} \otimes H_{2}$ if and only if $P_{1}$ occurs in $H_{1}$. Another way of describing Axiom 5 is that if $P_{1}$ is an event in system 1 and we test whether $P_{1}$ occurs in the combined system, then this test should not be affected by system 2 . This statement is made precise in the next lemma where $\bar{c}$ denotes the complex conjugate of $c \in \mathbb{C}$. Also, note that we use the physics convention that the inner product is anti-linear in the first argument.

Lemma 1. If $\gamma \in H_{1} \otimes H_{2}$ is a state and $P_{1} \in \mathcal{L}\left(H_{1}\right)$ is an event, then there exist states $\alpha_{i} \in H_{1}$ and $\lambda_{i} \in[0,1]$ with $\sum \lambda_{i}=1$ such that

$$
\left\langle\gamma, P_{1} \otimes I_{2} \gamma\right\rangle=\sum \lambda_{i}\left\langle\alpha_{i}, P_{1} \alpha_{i}\right\rangle
$$

Proof. The state $\gamma$ has the form (1) with $\sum\left|c_{i j}\right|^{2}=1$. We then have that

$$
\begin{aligned}
\left\langle\gamma, P_{1} \otimes I_{2} \gamma\right\rangle & =\left\langle\sum c_{i j} \phi_{i} \otimes \psi_{j}, P_{1} \otimes I_{2} \sum c_{r s} \phi_{r} \otimes \psi_{s}\right\rangle \\
& =\sum \bar{c}_{i j} \sum c_{r s}\left\langle\phi_{i} \otimes \psi_{j}, P_{1} \phi_{r} \otimes \psi_{s}\right\rangle \\
& =\sum_{i, j, r} \bar{c}_{i j} c_{r j}\left\langle\phi_{i}, P_{1} \psi_{r}\right\rangle
\end{aligned}
$$

Letting

$$
\alpha_{j}=\sum_{i} c_{i j} \phi_{i} / \sum_{i}\left|c_{i j}\right|^{2}
$$

and $\lambda_{j}=\sum_{i}\left|c_{i j}\right|^{2}$ we conclude that $\alpha_{j}$ are states in $H_{1}$, $\sum \lambda_{j}=1$ and that (2) holds.

Equation (2) says that there are states $\alpha_{i} \in H_{1}$ such that $\mathcal{P}_{\gamma}\left(P_{1} \otimes I_{2}\right)$ is a convex combination of $\mathcal{P}_{\alpha_{i}}\left(P_{1}\right)$. In this way, a test of the occurrence of $P_{1} \otimes I_{2}$ only depends on states in $H_{1}$ and is independent of system 2. The function $P_{1} \mapsto \sum \lambda_{i}\left\langle\alpha_{i}, P_{1} \alpha_{i}\right\rangle$ in (2) is called a mixed state [3,7] and we shall not pursue these further.

We now come to our main definition. A state $\gamma \in H_{1} \otimes H_{2}$ is factorized (or a product state) if $\gamma=\alpha \otimes \beta$ for states $\alpha \in H_{1}, \beta \in H_{2}$. Otherwise, $\gamma$ is entangled. When $\gamma=\alpha \otimes \beta$, we call $\alpha$ and $\beta$ the local parts of $\gamma$. The local parts are not unique because if $\alpha$ and $\beta$ are local parts, then so are $e^{i \theta} \alpha, e^{-i \theta} \beta$ where $\theta \in \mathbb{R}, i=\sqrt{-1}$. 
Example 1. If $\alpha, \beta$ are orthogonal states in $H$, then

$$
\delta=\frac{1}{\sqrt{2}}(\alpha \otimes \beta-\beta \otimes \alpha)
$$

is an entangled state in $H \otimes H$. To show this suppose that $\delta=\phi \otimes \psi$ for states $\phi, \psi \in H$. Taking the inner product with $\alpha \otimes \alpha$ gives $\langle\alpha, \phi\rangle\langle\alpha, \psi\rangle=0$ so $\langle\alpha, \phi\rangle=0$ or $\langle\alpha, \psi\rangle=0$. Suppose $\langle\alpha, \phi\rangle=0$ and take the inner product with $\alpha \otimes \beta$ to obtain

$$
\frac{1}{\sqrt{2}}=\langle\alpha, \phi\rangle\langle\beta, \psi\rangle
$$

which gives a contradiction. If $\langle\alpha, \psi\rangle=0$, take the inner product with $\beta \otimes \alpha$ to again get a contradiction. Hence $\delta$ is entangled.

\section{Action at a Distance}

Alice and Bob prepare an interacting pair of electrons in a state $\gamma$ at a lab in New York; Alice keeps her electron (system 1) in New York and Bob takes his electron (system 2) to a lab on the moon. Since all electrons have the same properties, both systems give a copy of a Hilbert space $H$ and the combined system has Hilbert space $H \otimes H$. For this experiment, $\gamma$ is the factorized state $\alpha \otimes \beta$ where $\alpha, \beta$ are orthogonal states in $H$. Let $P$ be an event that pertains to an electron (say, spin-up in the $z$-direction). Alice sends her electron through an apparatus that tests $P$. She confirms that $P$ occurs so by Axioms 3 and $5, \gamma$ updates to

$$
\gamma^{\prime}=\frac{P \otimes I(\alpha \otimes \beta)}{\|P \otimes I(\alpha \otimes \beta)\|}=\frac{P \alpha \otimes \beta}{\|P \alpha\|}
$$

It is fairly clear that Bob's electron is unaffected. To make sure, suppose Bob tests an event $Q$ pertaining to his electron. According to $\gamma$, we have

$$
\begin{aligned}
\mathcal{P}_{\gamma}(I \otimes Q) & =\langle\alpha \otimes \beta, I \otimes Q(\alpha \otimes \beta)\rangle \\
& =\langle\alpha \otimes \beta, \alpha \otimes Q \beta\rangle=\langle\beta, Q \beta\rangle
\end{aligned}
$$

Moreover, we obtain

$$
\begin{aligned}
\mathcal{P}_{\gamma^{\prime}}(I \otimes Q) & =\frac{1}{\|P \alpha\|^{2}}\langle P \alpha \otimes \beta,(I \otimes Q)(P \alpha \otimes \beta)\rangle \\
& =\frac{1}{\|P \alpha\|^{2}}\langle P \alpha \otimes \beta, P \alpha \otimes Q \beta\rangle=\langle\beta, Q \beta\rangle
\end{aligned}
$$

We conclude that Bob's electron is in the same state after Alice's measurement as it was before.

Next Bob returns to New York where he and Alice prepare a pair of electrons in the entangled state $\delta$ of (3). Alice keeps her electron in New York and Bob again takes his to the moon. Now Alice performs her experiment that tests $P$ and confirms that $P$ occurs. (It actually does not matter if $P$ occurs. If it does not, then $P^{\prime}$ occurs and our conclusion will be the same.) The updated state becomes

$$
\delta^{\prime}=\frac{(P \otimes I) \delta}{\|(P \otimes I) \delta\|}
$$

We have that

$$
\begin{aligned}
\|(P \otimes I) \delta\|^{2} & =\frac{1}{2}\langle P \alpha \otimes \beta-P \beta \otimes \alpha, P \alpha \otimes \beta-P \beta \otimes \alpha\rangle \\
& =\frac{1}{2}(\langle\alpha, P \alpha\rangle+\langle\beta, P \beta\rangle)
\end{aligned}
$$

Letting $N=\frac{1}{\sqrt{2}}(\langle\alpha, P \alpha\rangle+\langle\beta, P \beta\rangle)^{1 / 2}$ we obtain

$$
\delta^{\prime}=\frac{1}{N}(P \otimes I) \delta=\frac{1}{N}(P \alpha \otimes \beta-P \beta \otimes \alpha)
$$

It appears as if the state of Bob's electron is instantaneously changed without Bob doing anything. This is the spooky action at a distance that bothered Einstein and others. This effect in various forms really happens because it has been exhibited in thousands of experiments around the world. It does not violate special relativity which postulates that no signal or object can move faster than the speed of light. This is because further study shows that this action cannot relay any useable information or communication [1, 2, 8].

In order to be sure about this, let us show that the state of Bob's electron has indeed been changed. As before, let $Q$ be an event for Bob's electron. Before Alice made her measurement, the joint state was $\delta$ and the probability that $Q$ occurs is

$$
\begin{aligned}
\langle\delta, I \otimes Q \delta\rangle & =\frac{1}{2}\langle\alpha \otimes \beta-\beta \otimes \alpha, \alpha \otimes Q \beta-\beta \otimes Q \alpha\rangle \\
& =\frac{1}{2}(\langle\beta, Q \beta\rangle+\langle\alpha, Q \alpha\rangle)
\end{aligned}
$$

After Alice makes her measurement, the joint state is $\delta^{\prime}$ and the probability that $Q$ occurs becomes

$$
\begin{aligned}
\left\langle\delta^{\prime}, I \otimes Q \delta^{\prime}\right\rangle= & \frac{1}{N^{2}}(P \alpha \otimes \beta-P \beta \otimes \alpha, P \alpha \otimes Q \beta-P \beta \otimes Q \alpha) \\
=\frac{1}{N^{2}} & (\langle\alpha, P \alpha\rangle\langle\beta, Q \beta\rangle-\langle\alpha, P \beta\rangle\langle\beta, Q \alpha\rangle \\
& -\langle\beta, P \alpha\rangle\langle\alpha, Q \beta\rangle+\langle\beta, P \beta\rangle\langle\alpha, Q \alpha\rangle)
\end{aligned}
$$

We see that (4) and (5) are definitely different. As a simple example, suppose $P=Q=P_{\alpha}$ is the one-dimensional projection given by $P_{\alpha} \psi=\langle\alpha, \psi\rangle \alpha$ for all $\psi \in H$. Then (4) is $1 / 2$ while (5) is zero. We conclude that Alice's measurement on her electron has instantaneously altered Bob's electron. 


\section{Entangled or Not Entangled}

This section presents a simple criterion that determines whether a state is entangled or not [9].

Example 2. It is not so easy to tell whether a state is entangled. Let $\left\{\phi_{i}\right\},\left\{\psi_{i}\right\}, i=1,2,3$, be orthonormal bases for $H_{1}, H_{2}$, respectively and form the joint state $\psi \in H_{1} \otimes H_{2}$ given by

$$
\begin{aligned}
\psi=\frac{1}{N} & \left(4 \phi_{1} \otimes \psi_{1}-3 i \phi_{1} \otimes \psi_{2}+5 \phi_{1} \otimes \psi_{3}\right. \\
& -8 \phi_{2} \otimes \psi_{1}+6 i \phi_{2} \otimes \psi_{2}-10 \phi_{3} \otimes \psi_{3} \\
& \left.+12 \phi_{3} \otimes \psi_{1}-9 i \phi_{3} \otimes \psi_{2}+15 \phi_{3} \otimes \psi_{3}\right)
\end{aligned}
$$

where $i=\sqrt{-1}$ and $N=10 \sqrt{7}$ is the norm of the vector in parentheses. Is $\psi$ factorized and if it is, what are the local parts of $\psi$ ? If you can answer this outright, you are better than I. I prefer to use the following result [9,10].

Theorem 2. Let $\left\{\phi_{1}, \phi_{2}, \ldots, \phi_{m}\right\},\left\{\psi_{1}, \psi_{2}, \ldots, \psi_{n}\right\}$ be orthonormal bases for $\mathrm{H}_{1}, \mathrm{H}_{2}$, respectively and let

$$
\psi=\sum c_{i j} \phi_{i} \otimes \psi_{j} \in H_{1} \otimes H_{2}
$$

be a state, where $\sum c_{i j} \neq 0$. Then $\psi$ is factorized if and only if for all $i=1,2, \ldots, m, j=1,2, \ldots, n$ we have that

$$
c_{i j} \sum_{i, j} c_{i j}=\sum_{j} c_{i j} \sum_{i} c_{i j}
$$

Moreover, if $\psi$ is factorized, then the local parts are $\alpha /\|\alpha\|, \beta /\|\beta\|$ where $\alpha=\sum a_{i} \phi_{i}, \beta=\sum b_{j} \psi_{j}, a_{i}=$ $\frac{1}{c} \sum_{j} c_{i j}, b_{j}=\sum_{i} c_{i j}, c=\sum_{i j} c_{i j}$.

Proof. We know that $\psi$ is factorized if and only if $\psi=$ $\alpha \otimes \beta$ for some states $\alpha \in H_{1}, \beta \in H_{2}$. Let $\alpha=\sum a_{i} \phi_{i}$ and $\beta=\sum b_{j} \psi_{j}$. It follows that

$$
\sum_{i, j} c_{i j} \phi_{i} \otimes \psi_{j}=\left(\sum_{i} a_{i} \phi_{i}\right) \otimes\left(\sum_{j} b_{j} \psi_{j}\right)=\sum_{i, j} a_{i} b_{j} \phi_{i} \otimes \psi_{j}
$$

Hence, $\psi$ is factorized if and only if there exist sequences of complex numbers $\left\{a_{i}\right\},\left\{b_{j}\right\}, i=1,2, \ldots, m, j=$ $1,2, \ldots, n$ such that $c_{i j}=a_{i} b_{j}$ where $\sum\left|a_{i}\right|^{2}=\sum\left|b_{j}\right|^{2}=1$. If (6) holds, letting $c=\sum_{i, j} c_{i j}, a_{i}^{\prime}=\frac{1}{c} \sum_{j} c_{i j}, b_{j}^{\prime}=\sum_{i} c_{i j}$ we have that $c_{i j}=a_{i}^{\prime} b_{j}^{\prime}$. Since $\|\psi\|=1$ we have that

$$
\sum_{i}\left|a_{i}^{\prime}\right|^{2} \sum_{j}\left|b_{j}^{\prime}\right|^{2}=\sum_{i, j}\left|c_{i j}^{2}\right|=1
$$

Letting $a_{i}=a_{i}^{\prime} / \sqrt{\sum\left|a_{i}^{\prime}\right|^{2}}$ and $b_{j}=b_{j}^{\prime} / \sqrt{\sum\left|b_{j}^{\prime}\right|^{2}}$ we obtain $c_{i j}=a_{i} b_{j}$ and $\sum\left|a_{i}\right|^{2}=\sum\left|b_{j}\right|^{2}=1$. Hence, $\psi$ is factorized. Conversely, suppose that $\psi$ is factorized so there exist sequences $\left\{a_{i}\right\},\left\{b_{j}\right\}$ with $c_{i j}=a_{i} b_{j}$. We conclude that

$$
\sum_{j} c_{i j} \sum_{i} c_{i j}=a_{i} b_{j} \sum_{i, j} a_{i} b_{j}=c_{i j} \sum_{i, j} c_{i j}
$$

so (6) holds. The last sentence follows from our previous work.

Theorem 2 gives a necessary and sufficient condition for factorizability under the condition that $\sum c_{i j} \neq 0$. A more complicated criterion than (6) gives such a characterization in terms of the $c_{i j}$ without this condition [9]. Theorem 5(b) will give another characterization.

Example 3. We use Theorem 2 to answer the question in Example 2. Except for the common factor $1 / N$ we have that $c_{11}=4, c_{12}=-3 i, c_{13}=5, c_{21}=-8, c_{22}=6 i$, $c_{23}=-10, c_{31}=12, c_{32}=-9 i, c_{33}=15$. We then obtain $\sum_{i, j} c_{i j}=6(3-i), \sum_{j} c_{1 j}=3(3-i), \sum_{j} c_{2 j}=6(i-3)$, $\sum_{j} c_{3 j}=9(3-i), \sum_{i} c_{i 1}=8, \sum_{i} c_{i 2}=-6 i, \sum_{i} c_{i 3}=0$. It is easy to check that (6) holds (the factor $1 / N$ cancels from both sides). Also, the local parts become

$$
\begin{aligned}
& \alpha=\frac{1}{\sqrt{14}}\left(\phi_{1}-2 \phi_{2}+3 \phi_{3}\right), \\
& \beta=\frac{1}{5 \sqrt{2}}\left(4 \psi_{1}-3 i \psi_{2}+5 \psi_{3}\right) .
\end{aligned}
$$

The following Schmidt decomposition theorem [5--7] is important in this work.

Theorem 3. Any state $\psi \in H_{1} \otimes H_{2}$ has a Schmidt decomposition

$$
\psi=\sum_{i=1}^{r} \sqrt{\lambda_{i}} \phi_{i} \otimes \psi_{i}
$$

where $\lambda_{i}>0, \sum \lambda_{i}=1$ and $\left\{\phi_{i}\right\},\left\{\psi_{i}\right\}$ are orthonormal vectors in $H_{1}, H_{2}$, respectively.

In the Schmidt decomposition, the singular-values $\sqrt{\lambda_{i}}$ are unique, $i=1,2, \ldots, r$. It follows that $\psi$ is factorized if and only if $r=1$ in (7). Why not just use this to test whether $\psi$ is factorized? One reason is that the Schmidt decomposition can be difficult to construct. Another reason is that Theorem 2 generalizes to multipartite systems (more than two parts) where no Schmidt decomposition is available [9].

\section{An Entanglement Measure}

We now present a measure of entanglement. Using this measure, we can decide how entangled a state is and when one state is more entangled than another. If a state $\psi \in$ $H_{1} \otimes H_{2}$ has Schmidt decomposition $\psi=\sum_{i=1}^{r} \sqrt{\lambda_{i}} \phi_{i} \otimes \psi_{i}$, 
$\lambda_{i}>0, \sum \lambda_{i}=1$, then $\underline{\lambda}=\left(\lambda_{1}, \lambda_{2}, \ldots, \lambda_{r}\right)$ is a probability distribution. The entanglement number of $\psi$ is

$e(\psi)=\left[1-\sum_{i=1}^{r} \lambda_{i}^{2}\right]^{1 / 2}=\left[\sum_{i \neq j} \lambda_{i} \lambda_{j}\right]^{1 / 2}=\left[\sum_{i} \lambda_{i}\left(1-\lambda_{i}\right)\right]^{1 / 2}$

Relative to the distribution $\lambda$, the last expression in (8) shows that $e(\psi)$ is the average deviation of $\lambda$ from 1 . Notice that $\psi$ is factorized if and only if $e(\psi)=0$ which is an important property for an entanglement measure.

There are various justifications for the definition (8) [10]. One is that if the distribution $\lambda$ is peaked near 1 , then $e(\psi)$ should be near 0 and if $\lambda$ is spread fairly equally, then $e(\psi)$ should be large. For example, suppose $\psi$ has Schmidt decomposition

$$
\psi=\frac{\sqrt{99}}{10} \phi_{1} \otimes \psi_{1}+\frac{1}{10} \phi_{2} \otimes \psi_{2}
$$

Then $\underline{\lambda}=\left(\frac{99}{100}, \frac{1}{100}\right)$ and $\psi$ has the dominate factorized term $\frac{\sqrt{99}}{100} \phi_{1} \otimes \psi_{1}$ together with a very subordinate term $\frac{1}{10} \phi_{2} \otimes \psi_{2}$ so $e(\psi)$ should be small. Indeed,

$$
e(\psi)=\left[1-\left(\frac{99}{100}\right)^{2}-\left(\frac{1}{100}\right)^{2}\right]^{1 / 2} \approx 0.14
$$

We call $r$ in the Schmidt decomposition of $\psi$ the index of $\psi$ and write $n(\psi)=r$. We say that $\psi$ is maximally entangled with index $n(\psi)=r \geq 2$, if $\lambda_{i}=1 / r, i=$ $1,2, \ldots, r$. In this case, the distribution $\underline{\lambda}$ is uniformly spread so the entanglement should be large. The next result verifies this and is a standard calculus maximization problem whose proof we leave to the reader.

Theorem 4. $e(\psi) \leq\left[\frac{n(\psi)-1}{n(\psi)}\right]^{1 / 2}$ and equality is achieved if and only if $\psi$ is maximally entangled with index $n(\psi)$.

Example 4. Let $\left\{\phi_{1}, \phi_{2}, \phi_{3}\right\},\left\{\psi_{1}, \psi_{2}, \psi_{3}\right\}$ be orthonormal bases for $H_{1}, H_{2}$, respectively. Define the following states:

$$
\begin{aligned}
& \alpha=\frac{1}{\sqrt{2}} \phi_{1} \otimes \psi_{1}+\frac{1}{\sqrt{2}} \phi_{2} \otimes \psi_{2} \\
& \beta=\frac{1}{\sqrt{3}} \phi_{1} \otimes \psi_{1}+\frac{1}{\sqrt{3}} \phi_{2} \otimes \psi_{2}+\frac{1}{\sqrt{3}} \phi_{3} \otimes \psi_{3} \\
& \gamma=\frac{1}{\sqrt{2}} \phi_{1} \otimes \psi_{1}+\frac{1}{\sqrt{3}} \phi_{2} \otimes \psi_{2}+\frac{1}{\sqrt{6}} \phi_{3} \otimes \psi_{3} \\
& \delta=\frac{1}{3} \phi_{1} \otimes \psi_{1}+\frac{1}{3} \phi_{2} \otimes \psi_{2}+\sqrt{\frac{7}{9}} \phi_{3} \otimes \psi_{3}
\end{aligned}
$$

The distributions for these states are $\left(\frac{1}{2}, \frac{1}{2}\right),\left(\frac{1}{3}, \frac{1}{3}, \frac{1}{3}\right)$, $\left(\frac{1}{2}, \frac{1}{3}, \frac{1}{6}\right),\left(\frac{1}{9}, \frac{1}{9}, \frac{7}{9}\right)$, respectively. We see that $\alpha, \beta$ are maximally entangled with indexes 2,3 , respectively and $e(\alpha)=\frac{1}{\sqrt{2}}, e(\beta)=\sqrt{\frac{2}{3}}, e(\gamma)=\sqrt{\frac{11}{18}}, e(\delta)=\sqrt{\frac{30}{9}}$. Hence,

$$
e(\delta)<e(\alpha)<e(\gamma)<e(\beta)
$$

We mentioned in Section 3 that the Schmidt decomposition can be hard to compute. This is especially true for large index $r$ and is similar to finding the eigenvalues for an $r \times r$ matrix. We conclude that finding $e(\psi)$ using (8) can be quite difficult. We now give an efficient method for finding $e(\psi)$ that applies to any orthonormal basis $\left\{\phi_{i}\right\},\left\{\psi_{j}\right\}$ for $H_{1}, H_{2}$, respectively. Let $\psi=\sum c_{i j} \phi_{i} \otimes \psi_{j}$ and define the matrix $C=\left[c_{i j}\right]$. Denoting the adjoint of $C$ by $C^{*}$, we define the positive semidefinite, square matrix $|C|=\left(C^{*} C\right)^{1 / 2}$. For a square matrix $A=\left[a_{i j}\right]$, we define the trace of $A$ by $\operatorname{tr}(A)=\sum_{i} a_{i i}$.

Theorem 5. (a) $e(\psi)=\left[1-\operatorname{tr}\left(|C|^{4}\right)\right]^{1 / 2}$. (b) $\psi$ is factorized if and only if $\operatorname{tr}\left(|C|^{4}\right)=1$. (c) We have that

$$
\operatorname{tr}\left(|C|^{4}\right)=\sum_{r, s}\left[\left|\sum_{i} \bar{c}_{r i} c_{s i}\right|^{2}\right]
$$

Proof. (a) By the singular-value theorem [3], we can write $C=U D V$ where $U, V$ are unitary matrices and $D$ is a diagonal matrix

$$
D=\operatorname{diag}\left(\lambda_{1}^{1 / 2}, \lambda_{2}^{1 / 2}, \ldots, \lambda_{n}^{1 / 2}\right)
$$

with $\lambda_{i} \geq 0$ and $\sqrt{\lambda_{i}}$ are the singular-values of $C$. These singular-values coincide with those given in (7). Now

$$
|C|^{2}=C^{*} C=V^{*} D U^{*} U D V=V^{*} D^{2} V
$$

and hence, $|C|^{4}=V^{*} D^{4} V$. Therefore

$$
\sum \lambda_{i}^{2}=\operatorname{tr}\left(D^{4}\right)=\operatorname{tr}\left(|C|^{4}\right)
$$

We conclude from (8) that (a) holds. (b) follows from (a). To verify (c) we have that

$$
\begin{aligned}
|C|_{i j}^{4} & =\left(C^{*} C\right)\left(C^{*} C\right)_{i j}=\sum_{k}\left(C^{*} C\right)_{i k}\left(C^{*} C\right)_{k j} \\
& =\sum_{k, r} C_{i r}^{*} C_{r k} \sum C_{k s}^{*} C_{s j}=\sum_{r, s, k} \bar{c}_{r i} c_{r k} \bar{c}_{s k} c_{s j}
\end{aligned}
$$

Hence,

$$
\begin{aligned}
\operatorname{tr}\left(|C|^{4}\right) & =\sum_{i}|C|_{i i}^{4}=\sum_{i, k, r, s} \bar{c}_{r i} c_{r k} \bar{c}_{s k} c_{s i} \\
& =\sum_{r, s}\left[\sum_{i} \bar{c}_{r i} c_{s i} \sum_{k} \overline{\bar{c}_{r k} c_{s k}}\right]=\sum_{r, s}\left[\left|\sum_{i} \bar{c}_{r i} c_{s i}\right|^{2}\right] \square
\end{aligned}
$$

Example 5. Let $H$ be a 2-dimensional Hilbert space with orthonormal basis $\left\{\phi_{1}, \phi_{2}\right\}$ and let $\psi \in H \otimes H$ be the state

$$
\psi=\frac{1}{\sqrt{10}}\left(\phi_{1} \otimes \phi_{1}-2 i \phi_{1} \otimes \phi_{2}+\phi_{2} \otimes \phi_{1}-2 i \phi_{2} \otimes \phi_{2}\right)
$$

We then have that

$$
C=\frac{1}{\sqrt{10}}\left[\begin{array}{ll}
1 & -2 i \\
1 & -2 i
\end{array}\right]
$$


Applying Theorem 5 (c) gives

$$
\begin{aligned}
\operatorname{tr}\left(|C|^{4}\right)= & \sum_{r, s=1}^{2}\left|\bar{c}_{r 1} c_{s 1}+\bar{c}_{r 2} c_{s 2}\right|^{2} \\
= & \left(\left|c_{11}\right|^{2}+\left|c_{12}\right|^{2}\right)+\left(\left|c_{21}\right|^{2}+\left|c_{22}\right|^{2}\right) \\
& \quad+2\left|\bar{c}_{11} c_{21}+\bar{c}_{21} c_{22}\right|^{2} \\
= & \frac{1}{100}\left[(1+4)^{2}+(1+4)^{2}+2(1+4)^{2}\right]=1
\end{aligned}
$$

It follows from Theorem 5 (b) that $\psi$ is factorized. In fact, $\psi=\alpha \otimes \beta$ where $\alpha=\frac{1}{\sqrt{2}}\left(\phi_{1}+\phi_{2}\right), \beta=\frac{1}{\sqrt{5}}\left(\phi_{1}-2 i \phi_{2}\right)$.

Example 6. We change Example 5 slightly and let

$$
\psi=\frac{1}{\sqrt{10}}\left(\phi_{1} \otimes \phi_{1}-2 i \phi_{1} \otimes \phi_{2}+\phi_{2} \otimes \phi_{1}+2 i \phi_{2} \otimes \phi_{2}\right)
$$

We then have

$$
C=\frac{1}{\sqrt{10}}\left[\begin{array}{cc}
1 & -2 i \\
1 & 2 i
\end{array}\right]
$$

As in Example 5

$$
\operatorname{tr}\left(|C|^{4}\right)=\frac{1}{100}\left[(1+4)^{2}+(1+4) 2+2(1-4)^{2}\right]=\frac{17}{25}
$$

We conclude that $\psi$ is entangled with

$$
e(\psi)=\left(1-\frac{17}{25}\right)^{1 / 2}=\frac{2 \sqrt{2}}{5}
$$

In the previous two examples, we used Theorem 5 (c) to find $e(\psi)$. However, it is usually easier just to find $|C|^{4}$ directly and use Theorem 5 (a) as the following example shows.

Example 7. For $H$ defined as in Examples 5 and 6 , let $\psi=\frac{\sqrt{3}}{2} \phi_{1} \otimes \phi_{1}+\frac{1}{2 \sqrt{3}} \phi_{1} \otimes \phi_{2}+\frac{1}{2 \sqrt{3}} \phi_{2} \otimes \phi_{1}+\frac{1}{2 \sqrt{3}} \phi_{2} \otimes \phi_{2}$

The corresponding matrix becomes

$$
C=\frac{1}{2 \sqrt{3}}\left[\begin{array}{ll}
3 & 1 \\
1 & 1
\end{array}\right]
$$

Hence,

$$
|C|^{2}=\frac{1}{6}\left[\begin{array}{ll}
5 & 2 \\
2 & 1
\end{array}\right], \quad|C|^{4}=\frac{1}{36}\left[\begin{array}{cc}
29 & 12 \\
12 & 5
\end{array}\right]
$$

We conclude that $\operatorname{tr}\left(|C|^{4}\right)=17 / 18$ and hence,

$$
e(\psi)=\left(1-\frac{17}{18}\right)^{1 / 2}=\frac{1}{3 \sqrt{2}}
$$

\section{References}

[1] R. Horodecki, P. Horodecki, M. Horodecki, K. Horodecki. Quantum entanglement. Reviews of Modern Physics 2009; 81(2):865942. arXiv:quant-ph/0702225 doi: 10.1103/RevModPhys.81.865.

[2] L. M. Lederman, C. T. Hill. Quantum Physics for Poets. Prometheus Books, Amherst, New York, 2011.

[3] M. A. Nielsen, I. L. Chuang. Quantum Computation and Quantum Information. Cambridge University Press, Cambridge, 2010. doi:10.1017/ cbo9780511976667.

[4] S. Gudder. Quantum computation. The American Mathematical Monthly 2003; 110(3):181-201. doi: $10.1080 / 00029890.2003 .11919955$

[5] C. P. Williams, S. H. Clearwater. Explorations in Quantum Computing. Springer, New York, 1998.

[6] C. P. Williams. Explorations in Quantum Computing. 2nd Edition. Texts in Computer Science. Springer, London, 2011. doi:10.1007/978-184628-887-6.

[7] T. Heinosaari, M. Ziman. The Mathematical Language of Quantum Theory: From Uncertainty to Entanglement. Cambridge University Press, Cambridge, 2012. doi:10.1017/cbo9781139031103.

[8] P. Busch. The role of entanglement in quantum measurement and information processing. International Journal of Theoretical Physics 2003; 42(5):937-941. doi:10.1023/a:1025462220957.

[9] S. Gudder. A characterization for entangled vectors 2019; arXiv: 1902.08853

[10] S. Gudder. A theory of entanglement. Quanta 2020; 9(1):7-15. doi:10.12743/quanta.v9i1.115. 